\title{
Atmospheric coherence times in interferometry: definition and measurement
}

\author{
A. Kellerer ${ }^{1}$ and A. Tokovinin ${ }^{2}$ \\ ${ }^{1}$ European Southern Observatory, Karl-Schwarzschild-Strasse 2, 85748 Garching bei München, Germany \\ e-mail: akellere@eso.org \\ 2 Cerro Tololo Inter-American Observatory, Casilla 603, La Serena, Chile \\ e-mail: atokovinin@ctio.noao.edu
}

Received 9 June 2006 / Accepted 15 September 2006

ABSTRACT

\begin{abstract}
Aims. Current and future ground-based interferometers require knowledge of the atmospheric time constant $t_{0}$, but this parameter has diverse definitions. Moreover, adequate techniques for monitoring $t_{0}$ still have to be implemented.

Methods. We derive a new formula for the structure function of the fringe phase (piston) in a long-baseline interferometer, and review available techniques for measuring the atmospheric time constant and the shortcomings.

Results. It is shown that the standard adaptive-optics atmospheric time constant is sufficient for quantifying the piston coherence time, with only minor modifications. The residual error of a fast fringe tracker and the loss of fringe visibility in a finite exposure time are calculated in terms of the same parameter. A new method based on the fast variations of defocus is proposed. The formula for relating the defocus speed to the time constant is derived. Simulations of a 35-cm telescope demonstrate the feasibility of this new technique for site testing.
\end{abstract}

Key words. atmospheric effects - instrumentation: interferometers - site testing

\section{Introduction}

Astronomical sites for classical observations are characterized in terms of atmospheric image quality (seeing). For high-angular resolution techniques such as adaptive optics (AO) and interferometry, we need to know additional parameters. The atmospheric coherence time is one of these. Here we refine the definition of the interferometric coherence time, review available techniques, and propose a new method for its measurements.

The AO time constant, $\tau_{0}$, is a well-defined parameter related to the vertical distribution of turbulence and wind speed (Roddier 1981). To correct wave fronts in real time, a sufficient number of photons from the guide star is needed within each coherence area during time $\tau_{0}$. This severely restricts the choice of natural guide stars and tends to impose the complex use of laser guide stars (Hardy 1998). It is shown below that new, simple methods of $\tau_{0}$ monitoring are still needed.

Modern ground-based stellar interferometers attain extreme resolution, but their sensitivity is limited by the atmosphere. Even at the best observing sites, such as Paranal in Chile, fast fringe tracking is not fully operative yet, and one therefore tends to employ exposure times that are short enough to "freeze" the atmospheric turbulence. The price is a substantial loss in limiting magnitude. It is hence important to measure the time constant, $t_{0}$, of the piston $-i$. e. the mean phase over the telescope aperture - at existing and future sites. However, the exact definition of $t_{0}$ is not clear, any more than are methods to measure it. Do we need an interferometer to evaluate $t_{0}$ ? Is $t_{0}$ different from $\tau_{0}$ ? Does it depend on the aperture size and baseline? We review various definitions of the interferometric time constant based on the piston structure function (SF), on the error of a fringe tracker, and on the loss of fringe contrast during a finite exposure time.
It is shown that the piston time constant is proportional to the AO coherence time $\tau_{0}$, both depending on the same combination of atmospheric parameters.

During site exploration campaigns, one would like to predict the performance of large base-line interferometers, and it is desirable to do this with single-dish and, preferably, small telescopes. The existing techniques for $\tau_{0}$ measurement are listed and a new method for site testing proposed.

\section{Atmospheric coherence time in interferometry}

\subsection{Atmospheric coherence time $\tau_{0}$}

First, we introduce the relevant atmospheric parameters and the AO time constant $\tau_{0}$. For convenience, we outline the essential formulae, but for the general background, we refer the reader to Roddier (1981).

The spatial and temporal fluctuations of atmospheric phase distortion $\varphi$ are usually described by the SF

$D_{\varphi}(\boldsymbol{r}, t)=\left\langle\left[\varphi\left(\boldsymbol{r}^{\prime}, t^{\prime}\right)-\varphi\left(\boldsymbol{r}+\boldsymbol{r}^{\prime}, t+t^{\prime}\right)\right]^{2}\right\rangle$,

which depends on the transverse spatial coordinate $\boldsymbol{r}$ and time interval $t$. The angular brackets indicate statistical average.

The atmosphere consists of many layers. The contribution of a layer $i$ of thickness $\mathrm{d} h$ at altitude $h$ to the turbulence intensity is specified in terms of $C_{n}^{2}(h) \mathrm{d} h$, equivalently expressed through the Fried parameter $r_{0, i}^{-5 / 3}=0.423 k^{2} C_{n}^{2}(h) \mathrm{d} h, k=2 \pi / \lambda$ being the wavenumber. The spatial SF in the inertial range (between inner and outer scales) is

$D_{\varphi}(\boldsymbol{r}, 0)=6.883\left(|\boldsymbol{r}| / r_{0}\right)^{5 / 3}$. 
It is assumed that each layer moves as a whole with the velocity vector $\boldsymbol{V}(h)$ (Taylor hypothesis). The temporal SF of the piston fluctuations $D_{\varphi, i}(0, t)$ in one small aperture due to a single layer is then equal to the spatial SF at shift $V t$,

$D_{\varphi, i}(0, t)=6.883\left[V(h) t / r_{0, i}\right]^{5 / 3}$.

Summing the contributions of all layers, we obtain

$$
\begin{aligned}
D_{\varphi}(0, t) & =2.910 t^{5 / 3} k^{2} \int_{0}^{+\infty} V^{5 / 3}(h) C_{n}^{2}(h) \mathrm{d} h \\
& =6.883\left(t \bar{V}_{5 / 3} / r_{0}\right)^{5 / 3}=\left(t / \tau_{0}\right)^{5 / 3},
\end{aligned}
$$

where $\tau_{0}=0.314\left(r_{0} / \bar{V}_{5 / 3}\right)$ is the AO time constant (Roddier 1981) and the average wind speed $\bar{V}_{p}$ is computed as

$\bar{V}_{p}=\left[\frac{\int_{0}^{+\infty} V^{p}(h) C_{n}^{2}(h) \mathrm{d} h}{\int_{0}^{+\infty} C_{n}^{2}(h) \mathrm{d} h}\right]^{1 / p}$.

The formulae are valid for observations at zenith. At angle $\gamma$ from the zenith, the optical path is increased in proportion to the air mass, $\sec \gamma$, and the SF increases by the same factor. Further, the transverse component of the wind velocity changes. In the following, we neglect these complications and consider only observations at zenith, but the analysis of real data must account for $\gamma \neq 0$.

\subsection{Piston time constant}

In an interferometer with a large baseline $\left(B \gg L_{0}\right.$, where $L_{0}$ : turbulence outer scale) the phase patterns over the apertures are uncorrelated on short time scales. Thus, for a small time interval $(t<B / V)$, the SF of the phase difference $\phi$ (do not confuse with the phase $\varphi$ ) in an interferometer with two small apertures will simply be two times larger, $D_{\phi}(t)=2 D_{\varphi}(0, t)$ (Conan et al. 1995). As a result the differential piston variance reaches $1 \mathrm{rad}^{2}$ for a time delay $t_{0}=2^{-3 / 5} \tau_{0}=0.66 \tau_{0}$. Note that in the case of smaller baselines and large outer scales - when the assumption $B \gg L_{0}$ becomes invalid $-D_{\phi}(t)<2 D_{\varphi}(0, t)$ and the resulting coherence time, accordingly, lies between $0.66 \tau_{0}$ and $\tau_{0}$. Yet, $B \gg L_{0}$ applies to the characterization of large baseline interferometers at low-turbulence sites.

When an interferometer with larger circular apertures of diameter $d$ is considered, phase fluctuations are averaged inside each aperture. As shown later, for time increments smaller than $d / V$, the piston structure function is quadratic in $t$ and is essentially determined by the average wave-front tilt over the aperture. The variance of the gradient tilt $\alpha$ (in radians) in one direction is (Roddier 1981, Conan et al. 1995, Sasiela 1994)

$\sigma_{\alpha}^{2}=0.170 \lambda^{2} r_{0}^{-5 / 3} d^{-1 / 3}$

We write the piston $\mathrm{SF}$ in this regime as $D_{\phi}(t) \approx 2\left(k \sigma_{\alpha} V t\right)^{2}$, sum the contributions of all layers, and obtain the expression

$D_{\phi}(t) \approx 13.42\left(\bar{V}_{2} t / r_{0}\right)^{2}\left(r_{0} / d\right)^{1 / 3}=\left(t / t_{1}\right)^{2}$,

where the modified time constant $t_{1}=0.273\left(r_{0} / \bar{V}_{2}\right)\left(d / r_{0}\right)^{1 / 6}$. The analysis of the tilt variance with finite outer scale by Conan et al. (2000a) is applicable here. The finite outer scale reduces the amplitude of the tilt and hence increases the piston time constant, but this effect depends on the aperture size and is not very strong for $d<1 \mathrm{~m}$.

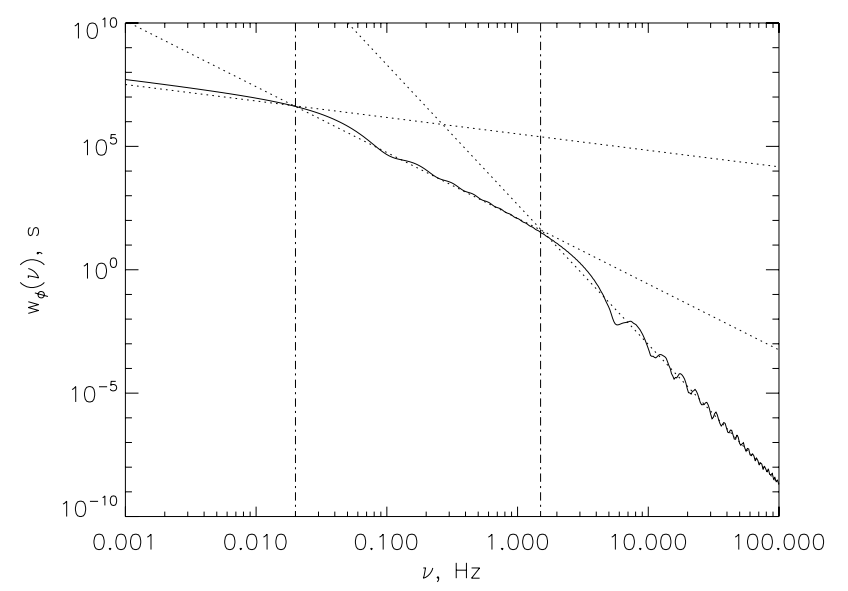

Fig. 1. Theoretical temporal power spectrum of the fringe position at $0.5 \mu \mathrm{m}$ wavelength. The two telescopes are separated by $100 \mathrm{~m}$ and have mirrors of $2 \mathrm{~m}$ diameter, the Fried parameter equals $r_{0}=11 \mathrm{~cm}$, the wind vector makes an angle of $\alpha=45^{\circ}$ with the baseline, $V=10 \mathrm{~m} / \mathrm{s}$. The vertical lines correspond to the frequencies: $0.2 \mathrm{~V} / \mathrm{B}$ and $0.3 \mathrm{~V} / \mathrm{d}$. The asymptotic power laws are $v^{-2 / 3}, v^{-8 / 3}, v^{-17 / 3}$ from lowest to highest frequencies.

Note that for small time intervals there is a weak dependence of the SF on the aperture diameter. Also, the wind velocity averaging is slightly modified. However, the expressions for $t_{1}$ and $t_{0}$ produce similar numerical results as long as $d / r_{0}$ is not too large. Thus, the system-independent definition of the AO time constant (4) also gives a good description of the temporal variations of the piston.

For time delays of approximately $B / V$ and larger, the pistons on two apertures are no longer independent. However, estimates of the time interval over which the Taylor hypothesis is valid range from $\sim 40 \mathrm{~ms}$ (Schoeck \& Spillar 1998) to several seconds (Colavita et al. 1987). Hence, at time intervals of $1 \mathrm{~s}$ or more, the Taylor hypothesis is insecure. Moreover, the finite turbulence outer scale reduces the amplitude of slow piston variations substantially. Here we concentrate only on rapid piston variations where our approximations are valid.

\subsection{Piston power spectrum and structure function}

The temporal power spectrum of the atmospheric fringe position has been derived by Conan et al. (1995). Their result is reproduced in Appendix A with minor changes. The temporal piston power spectrum (A.4) produced by a single turbulent layer is represented in Fig. 1 for a specific set of parameters. Because of the infinite outer scale $L_{0}$, this example is not realistic for frequencies below $\sim 1 \mathrm{~Hz}$. Moreover, as discussed in Sect. 2.2, Taylor's frozen flow hypothesis becomes invalid at low frequencies. Due to the infinite $L_{0}$, the asymptotic behavior of the spectrum, and in particular the cut-off frequencies, do not depend on the wind direction (Conan et al. 1995), whereas, in the real case of a finite outer scale, the cut-off frequencies are affected by wind direction, as described by Avila et al. (1997). Conan et al. (1995) point out that changing turbulence intensity and wind speed shift the spectrum vertically and horizontally, respectively, without changing the shape of the curve on the log-log plot. In observations with a small baseline $(\sim 12 \mathrm{~m})$, the proportionality to $v^{-2 / 3}$ at low frequencies and to $v^{-8 / 3}$ at medium frequencies has actually been measured, e.g. by Colavita et al. (1987). 


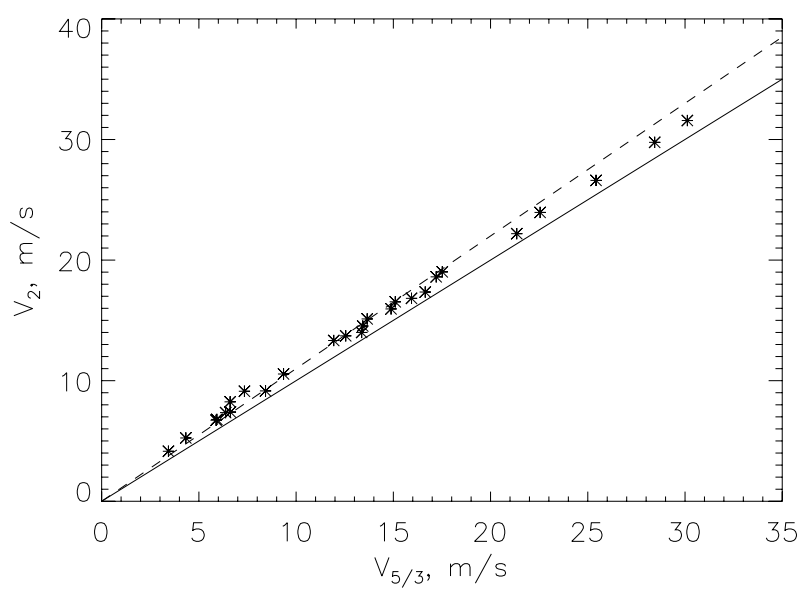

Fig. 2. Relation between average wind velocities $\bar{V}_{5 / 3}$ and $\bar{V}_{2}$ for 26 balloon profiles at Cerro Pachon in Chile (Avila et al. 2000). The full line corresponds to equality, the dashed line is $\bar{V}_{2}=1.1 \bar{V}_{5 / 3}$.

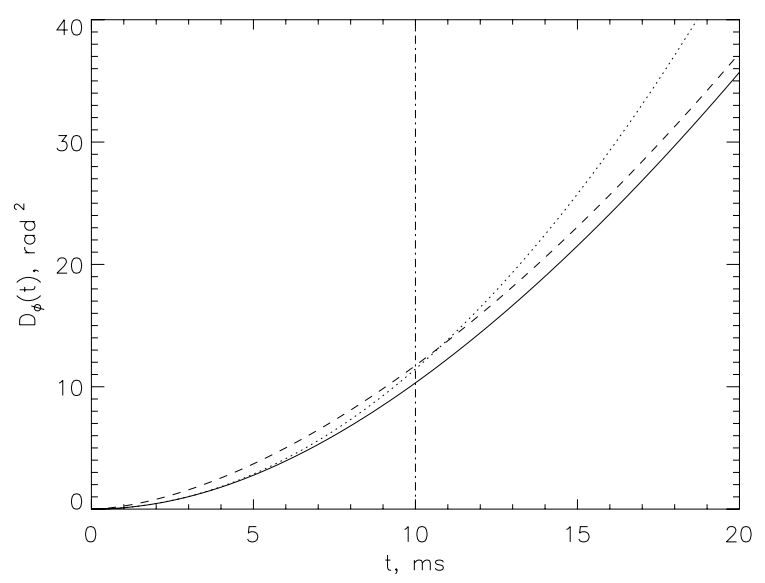

Fig. 3. Structure function of the fringe position for an interferometer with mirror diameters $d=0.1 \mathrm{~m}, r_{0}=11 \mathrm{~cm}, V=10 \mathrm{~m} / \mathrm{s}$. The vertical line corresponds to $t=d / V$. For $t<d / V$, the SF is quadratic in $\mathrm{t}$ (dotted line), cf. Eq. (7). For longer time scales, $D_{\phi} \approx 2 D_{\varphi}$ (dashed line).

Based on the piston power spectrum, we derive in Appendix A the new expression of the piston SF valid for time increments $t<\min \left(B / \bar{V}, L_{0} / \bar{V}\right)$ :

$D_{\phi}(t) \approx 13.76\left(\bar{V} t / r_{0}\right)^{2}\left[1.17\left(d / r_{0}\right)^{2}+\left(\bar{V} t / r_{0}\right)^{2}\right]^{-1 / 6}$.

As seen in Fig. 3, for $t>d / \bar{V}$, the piston averaging over apertures is not important and we obtain $D_{\phi}=2 D_{\varphi}$ in agreement with heuristic arguments. For very short increments $t \ll d / \bar{V}$, (8) reduces to (7). The average wind speed is $\bar{V} \approx \bar{V}_{5 / 3} \approx \bar{V}_{2}$. The difference between $\bar{V}_{5 / 3}$ and $\bar{V}_{2}$ is indeed small (Fig. 2).

\subsection{Error of a fringe tracking servo}

A fringe tracker measures the position of the central fringe and computes a correction. The actual compensation equals the integrated corrections applied after each iteration. Our analysis is similar to the classical work by Greenwood \& Fried (1976). For a more detailed model that takes the effect of the finite exposure and response times of the phasing device into account, see the work by Conan et al. (2000b). The error transfer function of a first-order phase-tracking loop equals

$T(v)=\mathrm{i} v /\left(v_{\mathrm{c}}+\mathrm{i} v\right)$,

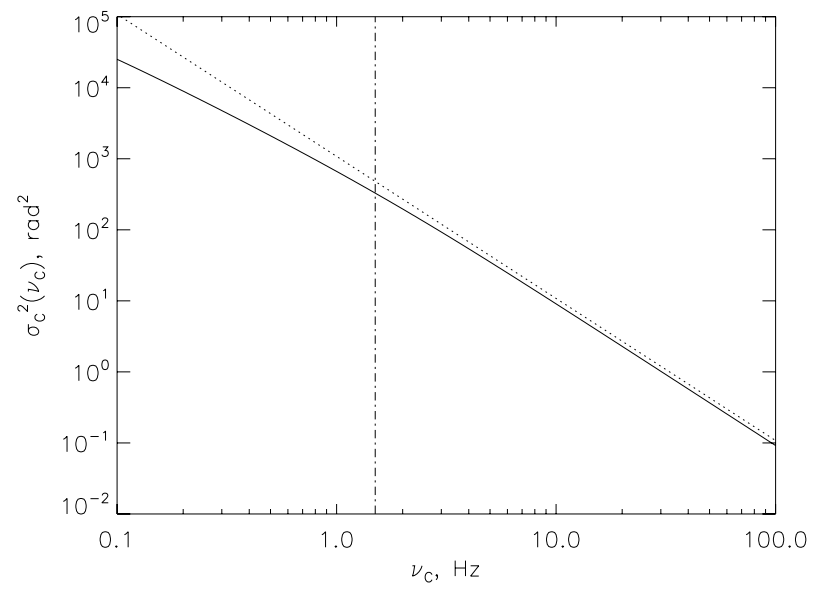

Fig. 4. Variance of corrected fringe position as a function of the bandwidth frequency of the correction system. The parameters of the simulation are identical to those of Fig. 1. At frequencies higher than $v_{\mathrm{c}}=0.3 \mathrm{~V} / \mathrm{d}$ (vertical line), the variance is approximated by $\left(2 \pi v_{\mathrm{c}} t_{1}\right)^{-2}$ (dotted line).

where $v_{\mathrm{c}}$ is the $3 \mathrm{~dB}$ bandwidth of the system. The temporal power spectrum of the corrected fringe position is $w_{\mathrm{c}}(v)=$ $|T(v)|^{2} w_{\phi}(v)$. The residual piston variance characterizes the performance of the phasing device. This variance is shown in Fig. 4 as a function of $v_{\mathrm{c}}$ and is given by

$\sigma_{\mathrm{c}}^{2}\left(v_{\mathrm{c}}\right)=\int_{-\infty}^{+\infty} v^{2} /\left(v_{\mathrm{c}}^{2}+v^{2}\right) w_{\phi}(v) \mathrm{d} v$.

When $v_{\mathrm{c}}<0.3 \mathrm{~V} / \mathrm{d}$, the fringe tracker is too slow and leaves a large residual error; only fast trackers with $v_{\mathrm{c}}>0.3 \mathrm{~V} / \mathrm{d}$ are of any practical interest. In this case, the dominant contribution to the residual variance in (10) comes from the frequencies just below $0.3 \mathrm{~V} / \mathrm{d}$, where the filter is approximated as $\left(v / v_{\mathrm{c}}\right)^{2}$. Hence the residual variance is proportional to the variance of the piston velocity. There is a simple relation between the residual error of the fringe tracker and the structure function of the piston. For small arguments $t$, we can replace $2[1-\cos (2 \pi v t)] \approx(2 \pi v t)^{2}$ in the expression (A.5) for the phase SF. Then the residual error of the fast fringe tracker is simply

$\sigma_{\mathrm{c}}^{2}\left(v_{\mathrm{c}}\right) \approx D_{\phi}\left[1 /\left(2 \pi v_{\mathrm{c}}\right)\right] \approx\left(2 \pi v_{\mathrm{c}} t_{1}\right)^{-2}$.

Thus, we have established that the error of the fast fringe tracker and the initial quadratic part of the piston SF are essentially determined by the variance of piston velocity which, in turn, depends on the tilt variance and the average wind speed $\bar{V}_{2}$.

\subsection{Summary of definitions and discussion}

Table 1 assembles different definitions of the atmospheric coherence time. We have demonstrated that the time constant $t_{0}$ of the piston SF is proportional to the AO time constant $\tau_{0}$. For small time increments, a slightly modified parameter $t_{1}$ should be used.

A different, but essentially equivalent, definition of the piston coherence time $T_{0}=0.81 r_{0} / \bar{V}_{5 / 3}=2.58 \tau_{0}$ has been given by Tango \& Twiss (1980) and reproduced by Colavita et al. (1987). It is the integration time during which the piston variance equals $1 \mathrm{rad}^{2}$. When fringes are integrated over a time $T_{0}$, the mean decrease in squared visibility equals $1 / e$. Here we use the more convenient definition $t_{0}=0.66 \tau_{0}$ based on the temporal SF and warn against confusion with Tango's $T_{0}$. The definition of $T_{0}$ is 
Table 1. Definitions of atmospheric time constants.

\begin{tabular}{lll}
\hline \hline Quantity of interest & Formula & Time constant \\
\hline Phase SF & $D_{\varphi}(t)=\left(t / \tau_{0}\right)^{5 / 3}$ & $\tau_{0}=0.314 r_{0} / \bar{V}_{5 / 3}$ \\
Piston SF, $t<d / V$ & $D_{\phi}(t)=\left(t / t_{1}\right)^{2}$ & $t_{1}=0.273\left(r_{0} / \bar{V}_{2}\right)\left(d / r_{0}\right)^{1 / 6}$ \\
Piston SF, $t>d / V$ & $D_{\phi}(t)=\left(t / t_{0}\right)^{5 / 3}$ & $t_{0}=0.66 \tau_{0}$ \\
Piston variance during an exposure $t>d / V$ & $\sigma_{\phi}^{2}(t)=\left(t / T_{0}\right)^{5 / 3}$ & $T_{0}=2.58 \tau_{0}$ \\
Phase tracker error, $v_{\mathrm{c}}>0.3 d / V$ & $\sigma_{\mathrm{c}}^{2}\left(v_{\mathrm{c}}\right)=\left(2 \pi v_{\mathrm{c}} t_{1}\right)^{-2}$ & $t_{1}$ \\
\hline
\end{tabular}

valid only for $T>d / V$, while shorter integration times are of practical interest (see below).

The performance of the fringe-tracker in a long-baseline interferometer can be characterized by the atmospheric time constant $t_{1}$ or, equivalently, by the average wind speed $\bar{V}_{2}$. The AO time constant $\tau_{0}$ (or $\bar{V}_{5 / 3}$ ) is also a good estimator of the piston coherence time, especially for small apertures $d \sim r_{0}$.

In order to reach a good magnitude limit, all modern interferometers have large apertures $d>r_{0}$. The atmospheric variance over the aperture is $1.03\left(d / r_{0}\right)^{5 / 3}>1 \mathrm{rad}^{2}$ and has to be corrected by some means (tip-tilt guiding, full AO correction, spatial filtering of the PSF) even at short integration times. The temporal piston variance will also be $>1 \mathrm{rad}^{2}$ on time scales of approximately $r_{0} / \bar{V}$ and longer. Hence exposure times shorter than $r_{0} / \bar{V}$ or fast fringe trackers are required in order to maintain high fringe contrast. In this regime, the relevant time constant that determines the visibility loss is $t_{1}$, rather than $\tau_{0}$ and $T_{0}$.

All definitions of atmospheric time constants contain a combination of $r_{0}$ and $\bar{V}$. As turbulence becomes stronger, the time constant decreases, although the wind speed may remain unchanged. Being less correlated, the parameters $r_{0}, \bar{V}$ are thus more suitable for characterizing atmospheric turbulence than the parameters $r_{0}, \tau_{0}$. Astronomical sites with "slow" or "fast" seeing should be ranked in terms of $\bar{V}$ rather than $\tau_{0}$. A fair correlation between $\bar{V}$ and the wind speed at $200 \mathrm{mB}$ altitude has been noted by Sarazin \& Tokovinin (2002).

\section{Measuring the atmospheric time constant}

\subsection{Existing methods of $\tau_{0}$ measurement}

Table 2 lists methods available for measuring the atmospheric coherence time $\tau_{0}$ or related parameters. The 3 rd column gives an indicative diameter of the telescope aperture required for each method. Short comments on each technique are given below.

SCIDAR (SCIntillation Detection And Ranging) has provided good results on $\tau_{0}$. It is not suitable for monitoring because manual data processing is still needed to extract $V(h)$, despite efforts to automate the process. Balloons provide only single-shot profiles of low individual statistical significance. The AO systems and interferometers give reliable results, but are not suitable for testing new sites or for long-term monitoring.

The methods listed in the next four rows of Table 2 all require small telescopes and can thus be used for site-testing. However, all these techniques have some intrinsic problems. SSS (Single Star SCIDAR) essentially extends the SCIDAR technique to small telescopes: profiles of $C_{n}^{2}(h)$ and $V(h)$ are obtained with lower height resolution than with the SCIDAR, and are then used to derive the coherence time. The GSM (Generalized Seeing Monitor) can only measure velocities of prominent layers after careful data processing. A coherence time, $\tau_{A A}-$ which,

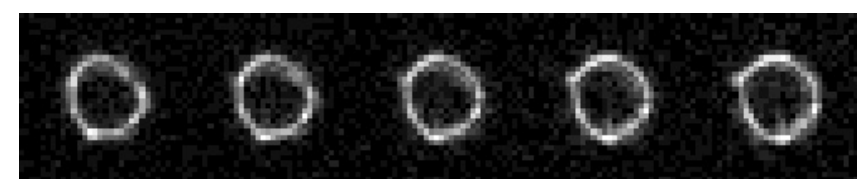

Fig. 5. Five consecutive ring images distorted by turbulence and detector noise. Each image is $16 \times 16$ pixels $\left(13.8^{\prime \prime}\right)$, the average ring radius is $3^{\prime \prime}$, the interval between images is $3 \mathrm{~ms}$, the wind speed is $10 \mathrm{~m} / \mathrm{s}$.

however, does not have a similar dependence on the turbulence profile than $\tau_{0}$ and $t_{1}-$ is deduced from the angle of arrival fluctuations. MASS (Multi-Aperture Scintillation Sensor) is a recent, but already well-proven, turbulence monitor. One of its observables related to scintillation in a $2 \mathrm{~cm}$ aperture approximates $\bar{V}_{5 / 3}$ (Tokovinin 2002), but this averaging does not include low layers and thus gives a biased estimate of $\tau_{0}$. An even less secure evaluation of $\tau_{0}$ can be obtained from DIMM (Differential Image Motion Monitor) by combining the measured $r_{0}$ with meteorological data on the wind speed (Sarazin \& Tokovinin 2002).

We conclude from this brief survey that a correct yet simple technique for measuring $\tau_{0}$ with a small-aperture telescope is still lacking. Such a method is proposed in the next section.

\subsection{The new method: FADE}

To measure the interferometric or AO time constant, we need an observable related to $\bar{V}_{2}$ or $\bar{V}_{5 / 3}$. The atmosphere consists of many layers with different wind speeds and directions, so a true $C_{n}^{2}$-weighted estimator (5) is required. Its response should be independent of the wind direction.

Wavefront distortions are commonly decomposed into Zernike modes (Noll 1976). The first mode, piston, cannot be sensed with a single telescope and the two subsequent modes, tip and tilt, tend to be corrupted by telescope vibrations. Of the remaining modes, the next three - defocus and two astigmatisms have the highest variance and are the best candidates for measuring atmospheric parameters.

The total turbulence integral (or $r_{0}$ ) is typically measured by the DIMM (Sarazin \& Roddier 1990). Lopez (1992) tried to derive $\tau_{0}$ from the speed of the DIMM signal, but this method did not prove to be practical. Because of its intrinsic asymmetry, DIMM does not provide an estimator of $\bar{V}$ that is independent of the wind direction. On the other hand, the fourth Zernike mode (defocus) is rotationally symmetric.

We show in Appendix B that the variance of defocus velocity provides an estimator of the time constant $t_{1}$. The variance of the defocus itself gives a measure of $r_{0}$. Thus, we can measure both $r_{0}$ and $\bar{V}_{2}$. The method is based on series of fast-defocus measurements, and we call it FADE (FAst DEfocus). The details of the future FADE instrument still need to be worked out and will be a subject of the forthcoming paper. Here we present 
Table 2. Methods of $\tau_{0}$ measurement.

\begin{tabular}{lllll}
\hline \hline Method & Measurables & $d, \mathrm{~m}$ & Problems & Reference \\
\hline SCIDAR & $C_{n}^{2}(h), V(h)$ & $>1$ & Needs large telescope & Fuchs et al. (1998) \\
Balloons & $C_{n}^{2}(h), V(h)$ & none & Expensive, no monitoring & Azouit \& Vernin (2005) \\
AO system & $r_{0}, \tau_{0}$ & $>1$ & Needs working AO & Fusco et al. (2004) \\
SSS & $C_{n}^{2}(h), V(h)$ & $>0.4$ & Low height resolution & Habib et al. (2006) \\
GSM & $r_{0}, V, \tau_{A A}$ & $4 \times 0.1$ & No obvious relation to $\tau_{0}$ and $t_{1}$ & Ziad et al. (2000) \\
MASS & $\tau_{0}^{*}$ & 0.02 & Biased (low layers ignored) & Kornilov et al. (2003) \\
DIMM & $r_{0}$ & 0.25 & Indirect $\tau_{0}$ estimate & Sarazin \& Tokovinin (2002) \\
FADE & $r_{0}, t_{1}$ & 0.35 & New method & This work \\
\hline
\end{tabular}
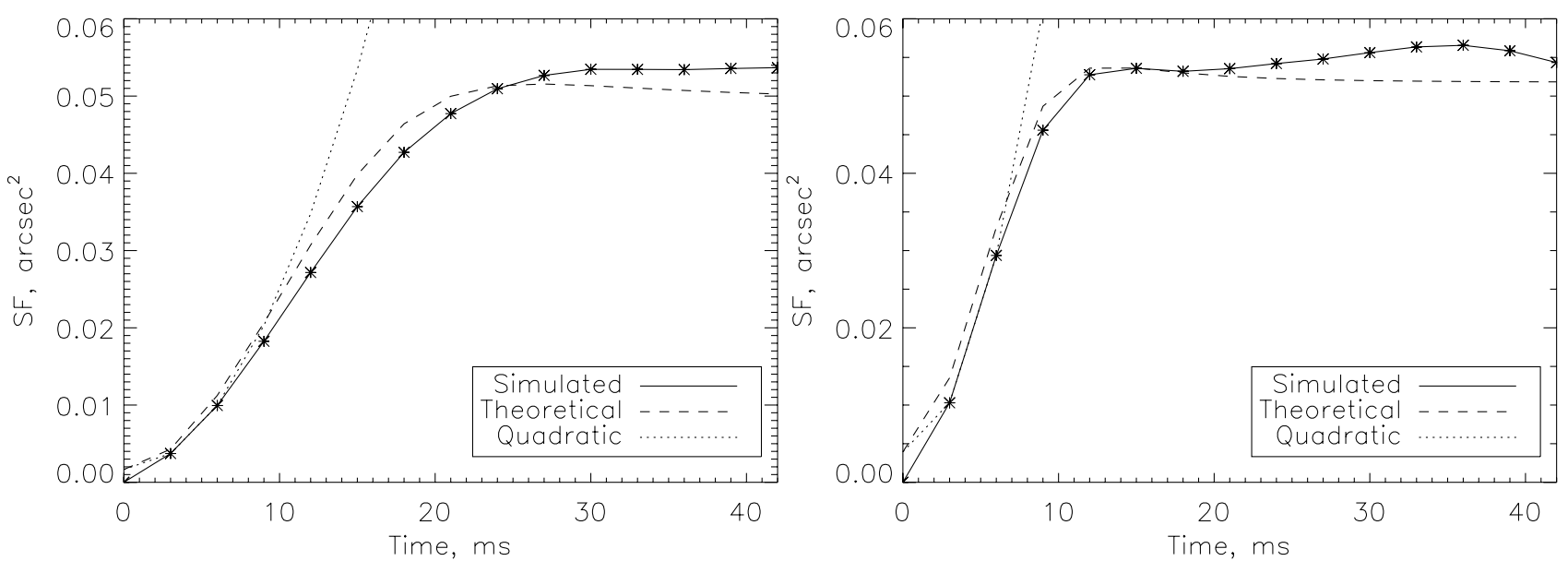

Fig. 6. Temporal structure functions of simulated measurements of the ring radius for wind speeds $10 \mathrm{~m} / \mathrm{s}($ left $)$ and $20 \mathrm{~m} / \mathrm{s}($ right $)$ and $r_{0}=0.1 \mathrm{~m}$ seeing (time constants $t_{1}$ of 3.36 and $1.68 \mathrm{~ms}$, respectively).

numerical simulations to show the feasibility of this approach. We simulated a telescope of $d=0.35 \mathrm{~m}$ diameter with a small central obstruction $\epsilon=0.1$. A conic aberration was introduced to form ring-like images (Fig. 5). This configuration resembles a DIMM with a continuous annular aperture. The ring radius $3^{\prime \prime}$ was chosen.

Monochromatic $(\lambda=500 \mathrm{~nm})$ images were computed on a $64^{2}$ pixel grid from the interpolated distortions and binned into CCD pixels of $0.86^{\prime \prime}$ size. We simulated photon noise corresponding to a star of $R=2$ magnitude and $3 \mathrm{~ms}$ exposure time (20000 photons per frame) and added a readout noise of 15 electrons rms in each pixel.

The radius $\rho$ of the ring image is calculated in the same way as standard centroids, by simply replacing coordinate with radius. The radius fluctuations $\Delta \rho$ serve as an estimator for the defocus coefficient $a_{4}$. The radius change is approximated by the average slope of the Zernike defocus between inner and outer borders of the aperture:

$\Delta \rho=C_{\rho} a_{4} \approx[2 \sqrt{3}(1+\epsilon) / \pi(\lambda / d)] a_{4}$.

The complex amplitude of the light distorted by two phase screens at 0 and $10 \mathrm{~km}$ altitude with combined $r_{0}=0.1 \mathrm{~m}$ was pre-calculated on a large square grid $(15 \mathrm{~m}$ size, $0.015 \mathrm{~m}$ pixels). This distribution is periodic in both coordinates, and it was "moved" in front of the aperture in a helical pattern with the wind speed $V$ to simulate the temporal evolution of the wavefront. The exposure time $\Delta t=3 \mathrm{~ms}$ corresponds to a wave-front shift $V \Delta t=0.06 \mathrm{~m}$ for $V=20 \mathrm{~m} / \mathrm{s}$, such that the initial quadratic part of the defocus SF $(\beta=2 V t / d<1)$ extends only to $\sim 3 \Delta t$.

Figure 6 shows the structure function, $D_{\rho}$, of the ring-image radius calculated from several seconds of simulated data. It contains a small additive component due to the measurement noise (in this case $0.05^{\prime \prime} \mathrm{rms}$ ), which was determined from the data itself by a quadratic fit to the 2 nd and 3rd points and its extrapolation to zero. The dashed lines are the theoretical SFs of defocus computed by (B.5) and converted into radius with the coefficient $C_{\rho}(12)$. The slope between the second and third points of the simulated SF closely matches the analytical formula.

To measure the speed of defocus variations, it is sufficient to fit a quadratic approximation to the initial part of the measured SF, $D_{\rho}(t) \approx a t^{2}$. Considering the noise, the best estimate of the coefficient $a$ is obtained from the second and third points, $a=\left[D_{\rho}(2 \Delta t)-D_{\rho}(\Delta t)\right] /\left(3 \Delta t^{2}\right)$. This estimator is not biased by white measurement noise. Equating the quadratic fit to the theoretical expression $D_{\rho}(t)=0.0269\left(C_{\rho} t / t_{1}\right)^{2}$, we get a recipe for calculating the time constant from the experimental data,

$t_{1} \approx 0.284 C_{\rho} \Delta t\left[D_{\rho}(2 \Delta t)-D_{\rho}(\Delta t)\right]^{-1 / 2}$.

Application of this formula to the simulated data gives $t_{1}$ values of 3.88 and $2.20 \mathrm{~ms}$ for wind speeds 10 and $20 \mathrm{~m} / \mathrm{s}$, while the input values are 3.36 and $1.68 \mathrm{~ms}$. Our simulated instrument slightly over-estimates $t_{1}$ because the chosen exposure time of $3 \mathrm{~ms}$ is too long. Indeed, the error gets worse for a higher wind speed and disappears for $V=5 \mathrm{~m} / \mathrm{s}$ (true and measured $t_{1}$ are 6.73 and $6.62 \mathrm{~ms}$ ) or for a shorter exposure time. In the real situation of a multi-layer atmosphere, the experimental SF will be the sum of the SFs produced by different layers. The contribution to the "jump" of the SF $D_{\rho}(2 \Delta t)-D_{\rho}(\Delta t)$ from fast layers will be reduced (in comparison with the quadratic formula) and will cause a bias in the measured $t_{1}$, increasing its value. 
The crudeness of our simulations (discrete shifts of the phase screen, approximate $C_{\rho}$, etc.) also contributes to the mismatch. Averaging of the image during finite exposure time has not been simulated yet. The response and bias of a real instrument will be studied thoroughly by a more detailed simulation. However, the feasibility of the proposed technique for measuring $t_{1}$ is already clear.

The next two Zernike modes number 5 and 6 (astigmatism) are not rotationally symmetric. However, the sum of the variances of the velocities of two astigmatism coefficients is again symmetric. In fact, it has the same spatial and temporal spectra as defocus, with a twice larger variance. Therefore, simultaneous measurement of the two astigmatism coefficients can be used to estimate the atmospheric time constant in the same way as defocus. Other measurables that are symmetric and have a cutoff at high frequencies can be used as well. However, defocus and astigmatism have the largest and slowest atmospheric variances making it easier to measure than other higher-order modes.

The FADE technique can be applied in a straightforward way to the analysis of the AO loop data, as a simple alternative to the more complicated method developed by Fusco et al. (2004).

\section{Conclusions}

We reviewed the theory of fast temporal variations in the phase difference in a large-baseline interferometer. For a practically interesting case of large apertures $d>r_{0}$, the piston SF usually exceeds $1 \mathrm{rad}^{2}$ at the aperture crossing time $t=d / \bar{V}$. Hence, shorter times are of interest where the piston SF is quadratic (rather than $\left.\propto t^{5 / 3}\right)$. The relevant atmospheric time constant is $t_{1}$. However, the standard AO time constant $\tau_{0}$ also provides a good estimation of the piston coherence time. Both these parameters essentially depend on the turbulence-weighted average wind speed $\bar{V}$.

A brief review of available methods for measuring $\tau_{0}$ shows the need for a simple technique suitable for site testing or monitoring, i.e. working on a small-aperture telescope. The FAst DEfocus (FADE) method proposed here fulfills this need. We argue that, for a given aperture size, this is the best way of extracting the information on $\tau_{0}$. The feasibility of the method is proven by simulation, which opens a way to the development of a real instrument. An instrument concept using a small telescope, some simple optics, and a fast camera will be described in a subsequent article.

\section{Appendix A: Derivation of the piston structure function}

The spatial power spectrum of the piston is derived from the spatial atmospheric phase spectrum (Roddier 1981)

$$
W_{\varphi}(\boldsymbol{f})=0.00969 k^{2} \int_{0}^{+\infty}\left(f^{2}+L_{0}^{-2}\right)^{-11 / 6} C_{n}^{2} \mathrm{~d} h
$$

where $f$ is the spatial frequency, $L_{0}$ the turbulence outer scale at height $h$, and the other notations were introduced in Sect. 2.1. We drop the explicit dependence of $C_{n}, L_{0}$, and all following altitude dependent-parameters on $h$, to ease the reading of the formulae. The spatial filter that converts $W_{\varphi}(\boldsymbol{f})$ into the piston power spectrum $W_{\phi}(\boldsymbol{f})$ is

$$
\begin{aligned}
M^{2}(\boldsymbol{f}) & =[2 \sin (\pi \boldsymbol{f} \boldsymbol{B}) A(\boldsymbol{f})]^{2} \\
W_{\phi}(\boldsymbol{f}) & =M^{2}(\boldsymbol{f}) W_{\varphi}(\boldsymbol{f}),
\end{aligned}
$$

for a baseline vector $\boldsymbol{B}$ and the aperture filter function $A(\boldsymbol{f})$. For a circular aperture of diameter $d, A(f)=2 J_{1}(\pi f d) /(\pi f d)$ and $f=|\boldsymbol{f}|$. There $J_{n}$ stands for the Bessel function of order $n$.

As usual, we assume that turbulent layers are transported with wind speed $\boldsymbol{V}$ directed at an angle $\alpha$ with respect to the baseline. The temporal power spectrum of the piston is then obtained by integrating in the frequency plane over a line displaced by $f_{x}=v / V$ from the coordinate origin and inclined at angle $\alpha$. Let $f_{y}$ be the integration variable along this line and $f^{2}=f_{x}^{2}+f_{y}^{2}$. The temporal spectrum equals

$$
\begin{aligned}
w_{\phi}(v)= & \frac{1}{V} \int_{-\infty}^{+\infty} W_{\phi}\left(f_{x} \cos \alpha+f_{y} \sin \alpha, f_{y} \cos \alpha-f_{x} \sin \alpha\right) \mathrm{d} f_{y} \\
= & 0.0388 k^{2} \int_{0}^{+\infty} V^{-1} C_{n}^{2} \mathrm{~d} h \int_{-\infty}^{+\infty}\left(f^{2}+L_{0}^{-2}\right)^{-11 / 6} \\
& \times\left[\sin \left(\pi B f_{x} \cos \alpha+\pi B f_{y} \sin \alpha\right) A(f)\right]^{2} \mathrm{~d} f_{y}
\end{aligned}
$$

We use the rotational symmetry of the aperture filter. This formula can be found in Conan et al. (1995) in a slightly different form. The temporal power spectrum is defined here on $v=(-\infty,+\infty)$ to keep the analogy with spatial power spectra.

The temporal structure function of the piston is

$D_{\phi}(t)=\int_{-\infty}^{+\infty} 2[1-\cos (2 \pi t v)] w_{\phi}(v) \mathrm{d} v$

For an interferometer with a large baseline $B \gg d$, the width of the aperture filter is much larger than the period of the $\sin ^{2}$ factor in (A.4). We can then replace the $\sin ^{2}$ with its average value 0.5 . Assuming also that $L_{0} \gg d$, we obtain an approximation for the piston power spectrum

$w_{\phi}(v) \approx 0.0194 k^{2} \int_{0}^{+\infty} V^{-1} C_{n}^{2} \mathrm{~d} h \int_{-\infty}^{+\infty} A^{2}(f) f^{-11 / 3} \mathrm{~d} f_{y}$.

With this approximation,

$$
\begin{aligned}
D_{\phi}(t)= & 0.0388 k^{2} \int_{0}^{+\infty} C_{n}^{2} \mathrm{~d} h \iint_{-\infty}^{+\infty}\left[1-\cos \left(2 \pi t f_{x} V\right)\right] \\
& \times A^{2}(f) f^{-11 / 3} \mathrm{~d} f_{x} \mathrm{~d} f_{y} \\
= & 0.244 k^{2} \int_{0}^{+\infty} C_{n}^{2} \mathrm{~d} h \int_{0}^{+\infty}\left[1-J_{0}(2 \pi t V f)\right] \\
& \times A^{2}(f) f^{-8 / 3} \mathrm{~d} f
\end{aligned}
$$

We used the relation (Gradshteyn \& Ryzhik 1965): $\int_{0}^{2 \pi} \cos (2 \pi z \cos \theta) \mathrm{d} \theta=2 \pi \quad J_{0}(2 \pi z)$. For a circular aperture of diameter $d$,

$D_{\phi}(t)=1.641 k^{2} d^{5 / 3} \int_{0}^{+\infty} C_{n}^{2} \mathrm{~d} h K_{1}(2 t V / d)$,

where the new dimensionless variables are $\beta=2 t V / d$ and $x=$ $\pi f d$ and the function $K_{1}(\beta)$

$$
\begin{aligned}
K_{1}(\beta) & =\int_{0}^{+\infty}\left[2 J_{1}(x) / x\right]^{2} x^{-8 / 3}\left[1-J_{0}(\beta x)\right] \mathrm{d} x \\
& \approx 1.1183 \frac{\beta^{2}}{\left(4.7+\beta^{2}\right)^{1 / 6}}
\end{aligned}
$$

The approximation of $K_{1}(\beta)$ is accurate to $1 \%$ for all values of the argument and reproduces the analytic solutions of the integral for very large and very small $\beta$. For example, for large $\beta$ the 
aperture filter tends to one; hence

$$
\begin{aligned}
K_{1}(\beta) & \approx \int_{0}^{\infty} x^{-8 / 3}\left[1-J_{0}(\beta x)\right] \mathrm{d} x \\
& =\pi /\left[2^{8 / 3} \Gamma^{2}(11 / 6) \sin (5 \pi / 6)\right] \beta^{5 / 3}=1.1183 \beta^{5 / 3}
\end{aligned}
$$

(cf. Eq. 20 in Noll 1976). It follows that for $t>d / \bar{V}$

$D_{\phi}(t) \approx 13.77\left(\bar{V}_{5 / 3} t / r_{0}\right)^{5 / 3}=\left(t / t_{0}\right)^{5 / 3}$.

For $t<d / \bar{V}, K_{1}(\beta) \approx 0.864 \beta^{2}$ and

$D_{\phi}(t) \approx 13.41\left(\bar{V}_{2} t / r_{0}\right)^{2}\left(r_{0} / d\right)^{1 / 3}=\left(t / t_{1}\right)^{2}$.

We recover (7). This proves that the initial part of the piston SF is indeed defined by the overall wavefront tilts.

For a single turbulent layer, the piston SF is directly proportional to $K_{1}(\beta)$. Considering the small difference between two alternative definitions of the average wind speed, $\bar{V}_{5 / 3} \approx \bar{V}_{2} \approx \bar{V}$, a good approximation for the SF at all time increments will be

$D_{\phi}(t) \approx 3.88\left(d / r_{0}\right)^{5 / 3} K_{1}(2 t \bar{V} / d)$.

With the approximation (A.9), we finally obtain (8).

\section{Appendix B: Fast focus variation}

The temporal power spectrum of the Zernike defocus coefficient $a_{4}$ is given in Conan et al. (1995) as

$w_{4}(v)=0.00969 k^{2} \int_{-\infty}^{+\infty} V^{-1} C_{n}^{2} \mathrm{~d} h \int_{-\infty}^{+\infty} A_{4}^{2}(f) f^{-11 / 3} \mathrm{~d} f_{y}$,

where $A_{4}(f)=2 \sqrt{3} J_{3}(\pi f d) /(\pi f d)$ is the spatial filter corresponding to the defocus on a clear aperture of diameter $d$ (Noll 1976), $f_{x}=v / V, f^{2}=f_{x}^{2}+f_{y}^{2}$, and we assume $L_{0} \gg d$. This expression is similar to (A.6) but has a two times smaller coefficient and a different aperture filter. The variance of defocus is a function of the Fried parameter:

$$
\begin{aligned}
\sigma_{4}^{2} & =\int_{-\infty}^{+\infty} w_{4}(v) \mathrm{d} v \\
& =0.00969 k^{2} \int_{0}^{+\infty} C_{n}^{2} \mathrm{~d} h \iint_{-\infty}^{+\infty} A_{4}^{2}(f) f^{-11 / 3} \mathrm{~d} f_{x} \mathrm{~d} f_{y} \\
& =0.0232\left(d / r_{0}\right)^{5 / 3} .
\end{aligned}
$$

The variance of the defocus velocity has the following dependence on atmospheric parameters:

$$
\begin{aligned}
S_{4}^{2}= & \int_{-\infty}^{+\infty}(2 \pi v)^{2} w_{4}(v) \mathrm{d} v \\
= & 0.383 k^{2} \int_{0}^{+\infty} V^{2} C_{n}^{2} \mathrm{~d} h \\
& \times \iint_{-\infty}^{+\infty} f_{x}^{2} A_{4}^{2}(f) f^{-11 / 3} \mathrm{~d} f_{x} \mathrm{~d} f_{y} .
\end{aligned}
$$

We set $x=\pi f d$ and find:

$$
\begin{aligned}
S_{4}^{2} & \approx 9.858 k^{2} d^{-1 / 3} \int_{0}^{+\infty} V^{2} C_{n}^{2} \mathrm{~d} h \int_{0}^{+\infty} J_{3}^{2}(x) x^{-8 / 3} \mathrm{~d} x \\
& =0.360\left(\bar{V}_{2} / r_{0}\right)^{2}\left(r_{0} / d\right)^{1 / 3}=0.0269 t_{1}^{-2} .
\end{aligned}
$$

The transformation from (B.3) to (B.4) involves a coefficient increase by $12 \pi^{2 / 3}$, while the definite integral is equal to $\Gamma(8 / 3) \Gamma(13 / 6) /\left[2^{8 / 3} \Gamma^{2}(11 / 6) \Gamma(29 / 6)\right]=0.01547$.

The SF of defocus $D_{4}(t)$ is derived in analogy with the piston SF, replacing the response $A_{1}(f)$ for piston with $A_{4}(f)$ for defocus. The coefficient is 2 times smaller because only one aperture is considered. In analogy with (A.8),

$$
D_{4}(t)=0.821 k^{2} d^{5 / 3} \int_{0}^{+\infty} C_{n}^{2} \mathrm{~d} h K_{4}(2 t V / d),
$$

$$
\begin{aligned}
K_{4}(\beta) & =12 \int_{0}^{+\infty}\left[J_{3}(x) / x\right]^{2} x^{-8 / 3}\left[1-J_{0}(\beta x)\right] \mathrm{d} x \\
& \approx \frac{0.0464 \beta^{2}+0.024 \beta^{6}}{1+1.2 \beta^{2}+\beta^{6}} .
\end{aligned}
$$

The approximation has a relative error less than $2 \%$ and correct asymptotes. Unlike $K_{1}$, the $K_{4}$ function saturates for large arguments. Considering only the initial quadratic part of $K_{4}$ at $\beta \ll 1$, we write for small time intervals

$D_{4}(t) \approx 0.360\left(t \bar{V}_{2} / r_{0}\right)^{2}\left(r_{0} / d\right)^{1 / 3}=0.0269\left(t / t_{1}\right)^{2}$.

\section{References}

Avila, R., Ziad, A., Borgnino, J., et al. 1997, J. Opt. Soc. Am. A., 14, 3070 Avila, R., Vernin, J., Chun M. R., et al. 2000, Proc. SPIE, 4007, 721 Azouit, M., \& Vernin, J. 2005, PASP, 117, 536

Colavita, M. M., Shao, M., \& Staelin, D. H. 1987, App. Opt., 26, 4106

Conan, J. M., Rousset, G., \& Madec, P. Y. 1995, J. Opt. Soc. Am. A, 12, 1559

Conan, R., Borgnino, J., Ziad, A., et al. 2000a, J. Opt. Soc. Am. A, 17, 1807

Conan, R., Ziad, A., Borgnino, J., et al. 2000b, proc. SPIE, 4006, 963

Fuchs, A., Tallon, M., \& Vernin, J. 1998, PASP, 110, 86

Fusco, T., Ageorges, N., Rousset, G., et al. 2004, proc. SPIE, 5490, 118

Gradshteyn, I. S., \& Ryzhik, I. M. 1965, Table of integrals, series, and products (New York: Academic Press), 5th ed.

Greenwood, D. P., \& Fried, D. L. 1976, J. Opt. Soc. Am., 66, 193

Habib, A., Vernin, J., Benkhaldoun, Z., \& Lanteri, H. 2006, MNRAS, 368, 1456 Hardy, J. W. 1998, Adaptive Optics for Astronomical Telescopes (Oxford: Oxford Univ. Press)

Kornilov, V., Tokovinin, A., Vozyakova, O., et al. 2003, Proc. SPIE, 4839, 837

Lopez, B. 1992, A\&A, 253, 635

Noll, R. 1976, J. Opt. Soc. Am., 66, 207

Roddier, F. 1981, Progress in Optics, 19, 281

Sasiela, R. J. 1994, Electromagnetic Wave Propagation in Turbulence (Berlin: Springer-Verlag)

Schoeck, M., \& Spillar, E. J. 1998, SPIE, 3353, 1092

Sarazin, M., \& Roddier, F. 1990, A\&A, 227, 294

Sarazin, M., \& Tokovinin, A. 2002, in Beyond conventional adaptive optics, ed. E. Vernet, R. Ragazzoni, S. Esposito, \& N. Hubin, ESO Conf. Workshop Proc. No. 58, Garching: ESO

Tango, W. J., \& Twiss, R. Q. 1980, Progress in Optics, XVII, 239

Tokovinin, A. 2002, Appl. Opt., 41, 957

Ziad, A., Conan, R., Tokovinin, A., et al. 2000, Appl. Opt., 39, 5415 